\title{
DETECTION OF EVOLUTION OF THE NUCLEUS OF NGC 2392
}

\author{
SARA R. HEAP \\ Laboratory for Astronomy and Solar Physics, Code 681, Goddard Space Flight Center, \\ Greenbelt MD 20771, U.S.A
}

\begin{abstract}
Ultraviolet spectra taken with the IUE satellite indicate that the central star of the planetary nebula, NGC 2392, has declined in brightness by $7 \%$ per decade. We interpret this fading as a consequence of evolution toward higher temperature. With this interpretation, the rate of optical fading suggests that the stellar mass is $0.73 M_{\odot}$.
\end{abstract}

Recently, two independent studies concluded that the nucleus of NGC 2392 is a relatively massive central star. From a non-LTE spectroscopic analysis, McCarthy et al. (1990) derived a stellar mass, $M=0.77(+.10,-.03) M_{\odot}$. In the second study, Pauldrach et al. (1988) showed that the anomalously low terminal velocity of the wind from the central star $\left(\mathrm{V}_{\infty}=500 \mathrm{~km} \mathrm{~s}^{-1}\right.$, Heap 1986) can be matched by theoretical models only if the star has a mass as high as $0.87 \pm 0.15 M_{\odot}$.

Stars this massive should evolve to higher temperatures quite rapidly. Blöcker and Schönberner's (1990) evolutionary models indicate that the central star should increase in temperature by $243{ }^{\circ} \mathrm{K}$ per year if $M=0.77 M_{\odot}$ and $928^{\circ} \mathrm{K}$ per year if $M=0.87 M_{\odot}$. Are such changes detectable by observation, and if so, how? Looking for direct signs of a temperature increase is a relatively coarse approach. A more sensitive test is optical fading. Since a central star evolves to higher temperatures at a constant bolometric luminosity, a larger fraction of its luminosity is emitted in the unobservable extreme ultraviolet, and a smaller fraction is emitted in the optical region of the spectrum.

Unfortunately, published estimates of stellar magnitude have been obtained in a variety of ways and are thus, not inter-comparable. Comparing ultraviolet fluxes measured by the IUE satellite is presently the most reliable way to detect optical fading in the central star of NGC 2392. The sensitivity of the short-wave spectrograph has been relatively stable and is well calibrated over its 14-year lifetime. IUE observations of NGC 2392 indicate that the flux from the central star has decreased by about $7 \%$ per decade in the wavelength interval, $1400-1900 \AA$. The decline shows no significant wavelength dependence, which is to be expected, since this spectral region is on the Rayleigh-Jeans tail of the blackbody curve. The decline in apparent brightness over a decade corresponds to a $0.73 M_{\odot}$ star.

\section{References}

Blöcker, T. \& Schönberner, D. 1990 A \& A, 240, L11

Heap, S. R.: 1986 ESA SP-263, p. 291

McCarthy, J. et al.: 1990 Ap.J., 351, 230

Pauldrach, A. et al.: 1988 A \& A, 207, 123 